\title{
Pulmonary Sarcoidosis with Associated Bloody Pleurisy
}

\author{
Megumi Watarai, Masanobu Yazawa, Ken Yamanda*, Hiroshi Yamamoto** and Yoshitaka Yamazaki***
}

\begin{abstract}
A 64-year-old man was admitted to our hospital complaining of non-productive cough and right chest pain. Chest radiographs showed bilateral hilar lymphadenopathy, diffuse granular nodules and right pleural effusion. Serum angiotensin-II-converting enzyme and lysozyme levels were elevated. Since thoracentesis indicated bloody pleurisy, video-assisted thoracoscopy was performed and revealed multiple white nodules on both the visceral and parietal pleura. Resected pleural biopsy specimens showed non-caseous granulomas. Furthermore, some nodules were observed to compress and involve small vessels and capillaries. The bloody pleurisy was assumed to have been derived from the rupture of small vessels that had been compressed and affected by the granuloma with sarcoidosis. (Internal Medicine 41: 1021-1023, 2002)
\end{abstract}

Key words: bronchoscopy, granuloma, thoracoscopy

\section{Introduction}

The characteristic findings of sarcoidosis are mainly bilateral hilar lymphadenopathy (BHL) and pulmonary granular opacities on chest images, with pleurisy being an unusual finding (1-3). However, we encountered a case of sarcoidosis with right bloody pleurisy; to investigate the cause of the hemorrhage, we performed video-assisted thoracoscopy (VATS).

\section{Case Report}

A 64-year-old Japanese man was admitted to our hospital in November 1999 because of non-productive cough and right chest pain which he had been suffering from for one month. His chest radiograph had appeared normal at a health checkup held at his company in June 1999, and he had had no previous disease. He had smoked 20 cigarettes per day for 30 years. On admission, the patient was $156 \mathrm{~cm}$ in height and weighed
$56 \mathrm{~kg}$; his temperature was 36.9 degrees Celsius, blood pressure 120/84 $\mathrm{mmHg}$ and heart rate 90 beats/min (regular). There was no superficial lymphadenopathy. Cardiac examination was entirely normal and auscultation of the lungs revealed decreased right breath sounds and friction rub. There was no abnormal finding of the abdomen, extremities, nervous system or skin. There was no finding suggestive of uveitis. Abnormal laboratory examination on admission produced the following results: angiotensin-II-converting enzyme (ACE), 22.7 IU/l (8.3-21.4); lysozyme, $18.6 \mathrm{ug} / \mathrm{ml}(5.0-10.2)$; soluble IL-2 receptor, 2,660 $\mathrm{U} / \mathrm{ml}$ (220-530). A tuberculin skin test was negative. Arterial blood gas analysis under room air revealed: $\mathrm{PO}_{2}, 73.0$ Torr; $\mathrm{PaCO}_{2}, 33.7$ Torr; $\mathrm{pH}, 7.46$. A chest radiograph showed BHL, diffuse pulmonary nodular infiltrates and right pleural effusion (Fig. 1). Thoracentesis of the right chest revealed bloody fluid with total protein $6.7 \mathrm{~g} / \mathrm{dl}$, lactate dehydrogenase (LDH) $1,103 \mathrm{IU} / \mathrm{l}$ and class II cytology. The adenosine deaminase activity (ADA) in the pleural effusion was not significantly elevated. Cultures of the pleural effusion and pleural tissues were negative for bacteria, as well as for mycobacteria. From this evidence, the pleurisy was suspected to be a hemothorax. A 67 gallium scintigram showed accumulation in the bilateral hilus and in the bilateral lung base. Bronchoscopic findings included a reticular arrangement of vessels on the carina and bilateral main bronchus, while transbronchial lung biopsy (TBLB) revealed non-caseous epithelioid cell granuloma compatible with sarcoidosis. However, as it is difficult to explain the occurrence of bloody pleurisy in patients with sarcoidosis, we thought it necessary to perform thoracoscopy in order to exclude diseases associated with bloody pleurisy, including malignancies. This was done on February 21, 2000. The thoracoscopy revealed many gray-white nodules on both the visceral and parietal pleura as well as on the diaphragm, and some nodules could be seen linked together like a bead necklace on the pleura (Fig. 2). Resected pleural specimens exhibited non-caseous epithelioid cell granulomas compatible with sarcoidosis, with some of the granulomas involving small vessels and capillaries, and showing signs of hemorrhage (Fig. 3). Since the patient's symptoms, including shortness of breath, general fatigue and pulmopleural involvement, continued for four months after the tho-

From the Department of Internal Medicine, Fujimi-Kogen Hospital, Fujimi-machi, *the Department of Thoracic Surgery, Chushin Matsumoto National Sanatorium, Matsumoto, **the First Department of Internal Medicine and ***the Department of Endoscopy, Shinshu University School of Medicine, Matsumoto

Received for publication January 23, 2002; Accepted for publication August 2, 2002

Reprint requests should be addressed to Dr. Yoshitaka Yamazaki, the Department of Endoscopy, Shinshu University School of Medicine, 3-1-1 Asahi, Matsumoto $390-8621$ 


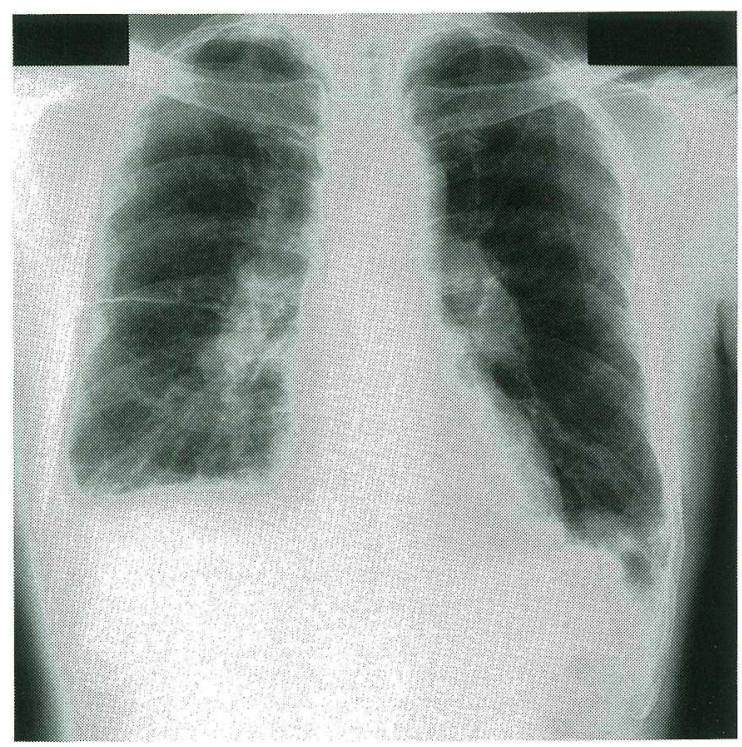

Figure 1. Chest radiograph revealed bilateral hilar lymphadenopathy, diffuse pulmonary nodular infiltrates and right pleural effusion.

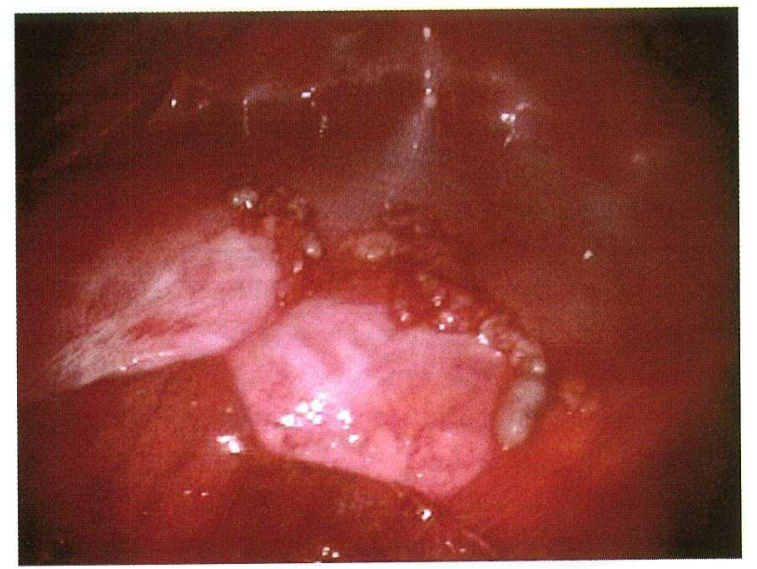

Figure 2. Thoracoscopy showed many gray-white nodules on both the visceral and parietal pleura, as well as on the diaphragm. Some of the nodules on the pleura appeared to be linked together like a bead necklace.

racoscopy, he was started on corticosteroid therapy $(0.5 \mathrm{mg} / \mathrm{kg}$ prednisolone) on March 31, 2000. The symptoms, BHL and pleural effusion in chest radiographs improved with therapy in about two weeks. Prednisolone was tapered off by $5 \mathrm{mg}$ every two weeks and was stopped in June 2000. There has been no recurrence of either signs or symptoms of right pleurisy.

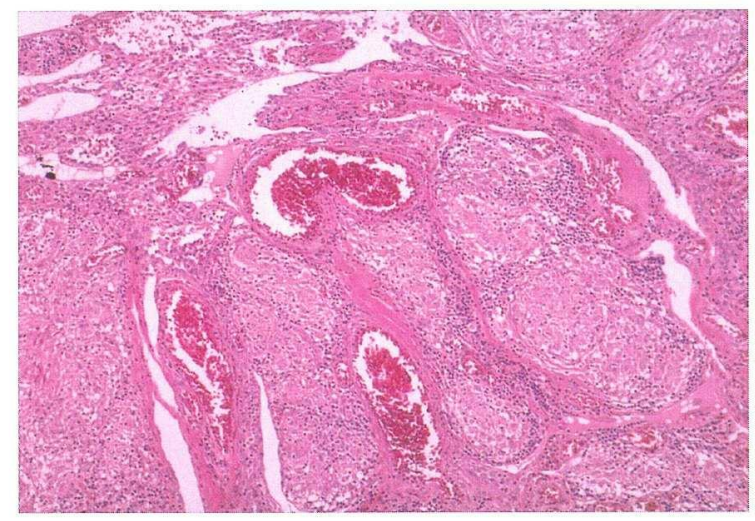

Figure 3. Resected specimens of the pleura exhibited noncaseous epithelioid cell granulomas compatible with sarcoidosis. Some of the granulomas were compressed and involved small veins, resulting in mildly venous congestion.

\section{Discussion}

The features of this case were compatible with sarcoidosis with BHL and an elevated serum ACE as well as elevated lysozyme levels and non-caseous granuloma (as confirmed by the TBLB). However, this did not adequately explain the existence of unilateral bloody pleurisy. Previous reports of pleurisy associated with sarcoidosis are relatively rare. Wilen et al (1) and Chusid and Siltzbach (2) reported having observed only 15 out of 227, and 7 out of 950 patients with sarcoidosis, respectively. In Japan, Fujii et al reported the disease in only 3 out of 64 sarcoidosis patients (3). Soskel and Sharma described the characteristics of pleurisy associated with sarcoidosis by saying that most effusions are small or moderate in size and are lymphocytic exudates (30-88\% lymphocytes) (4). It is believed that there are two mechanisms of pleurisy in patients with sarcoidosis one is inflammation of visceral and parietal pleura caused by peripheral lung granulomas, the other is the disturbance of venous and lymphatic circulation, which may result from BHL, in the peripheral vein and lymph duct (4). The occurrence of pleurisy in patients with sarcoidosis is therefore thought to be correlated with an exacerbated involvement of the lung parenchyma and BHL (5).

The occurrence of bloody pleurisy with sarcoidosis is even rarer. In fact, we know of only two cases of bloody pleurisy in such patients. In 1979, De Vuyst et al (6) reported a patient with sarcoidosis who had bilateral pleuritic pain, extensive parenchymal involvement and bloody pleural effusion. Over a decade later, Takahashi et al (7) reported bilateral bloody pleurisy, bloody ascites and hepatosplenomegaly in a case with sarcoidosis complicated by Heerfordt's syndrome. Using laparoscopy, they observed multiple patches of white plaque on the surface of the liver. The biopsy specimens showed giant cells and the involvement of lymphocytes but no liver cirrhosis or malignancies. Both cases were negative in terms of bacte- 


\section{Sarcoidosis with Bloody Pleurisy}

rial culture, tuberculosis and malignancy. Since corticosteroid therapy resulted in the rapid disappearance of the pleural effusion and progressive clearing of the parenchymal infiltrates, both groups supposed the cause of the bloody pleurisy to be pleural involvement of the sarcoidosis.

In the current case, it was necessary to exclude co-existing bacterial, tuberculous infection or malignancies using thoracoscopy. In fact, malignant lymphoma, mesothelioma and lung cancer are sometimes seen in patients with sarcoidosis $(8,9)$. We observed white plaque on the parietal and visceral pleura, a bloody effusion and coagulated blood, as well as many dilated small vessels around the white plaque on the surface of both pleura. The microscopic examination of resected specimens showed that some non-caseous granulomas involved neighboring small vessels and signs of hemorrhage on both pleura. Therefore, we suspected that the bloody pleurisy might have been derived from vessels which were involved and compressed by the granulomas. Further, chronic cough, which was induced by the bronchial lesion associated with sarcoidosis, might be one of the triggers of hemothorax.

Corticosteroids have been reported to be effective in the treatment of pleurisy with sarcoidosis, but cases of spontaneous resolution have also been reported (10-12). In our case, corticosteroid therapy resulted in marked improvement of the pleurisy as well as the parenchymal infiltrates, a result consistent with the inducement of bloody pleurisy being associated with granulomas disrupting small vessels.

In summary, we have described a case of sarcoidosis with associated bloody pleurisy. The derivation of bloody pleurisy was unclear, but it was most likely the result of the rupture of small vessels which were compressed and/or affected by the granuloma of sarcoidosis.

\section{References}

1) Wilen SB, Rabinowitz JG, Ulreich S, Lyons HA. Pleural involvement in sarcoidosis. Am J Med 57: 200-209, 1974.

2) Chusid EL, Siltzbach LE. Sarcoidosis of the pleura. Ann Intern Med 81: 190-194, 1974.

3) Fujii K, Yamamoto M, Kawazoe D, Nomura Y, Ogura Y. Pleural reaction in sarcoidosis. Nihon Kyobu Shikkan Gakkai Zasshi 10: 441-449, 1972.

4) Soskel NT, Sharma OP. Pleural involvement in sarcoidosis. Curr Opin Pulm Med 6: 455-468, 2000.

5) Salazar A, Mana J, Corbella X, Vidaller A. Sarcoid pleural effusion: a report of two cases. Sarcoidosis 11: 135-137, 1994.

6) De Vuyst P, De Troyer A, Yernault JC. Bloody pleural effusion in a patient with sarcoidosis. Chest 76: 607-609, 1979.

7) Takahashi N, Enomoto T, Hagiwara T, et al. A case of sarcoidosis presenting with Heerfordt's syndrome, associated with hepatosplenomegaly, pleural effusion, and ascites. Nihon Kyobu Shikkan Gakkai Zasshi 30: 684-688, 1992.

8) Brincker $\mathrm{H}$, Wilbek $\mathrm{E}$. The incidence of malignant tumors in patients with respiratory sarcoidosis. Brit J Cancer 29: 247-251, 1974.

9) Matsuzawa K, Hamada K, Tokuyama T, et al. Photomicrographs of a pleural biopsy specimens, showing malignant mesothelioma and necrosis-malignant pleural mesothelioma in a patient with sarcoidosis. Nihon Kyobu Shikkan Gakkai Zasshi 35: 687-691, 1997.

10) Sharma OP, Gordonson J. Pleural effusion in sarcoidosis: a report of six cases. Thorax 30: 95-101, 1975.

11) Nicholls AJ, Friend JA, Legge JS. Sarcoid pleural effusion: three cases and review of the literature. Thorax 35: 277-281, 1980.

12) Mizushima $Y$, Sassa K, Fujishita $T$, Iwai $H$, Yasumura $S$, Kobayashi $M$. Sarcoidosis accompanied by pleural effusion and multiple bronchial stenoses. Intern Med 36: 637-639, 1997. 